\title{
COMPARATIVO DE ÓBITOS, INTERNAÇÕES E LETALIDADE ENTRE 2019 E 2020 PARA CAUSAS SELECIONADAS NO BRASIL: UM ESTUDO DO POSSÍVEL IMPACTO DA PANDEMIA PELO NOVO CORONAVÍRUS
}

\author{
COMPARISON OF DEATHS, HOSPITALIZATIONS AND CASE FATALITY RATES BETWEEN 2019 AND 2020
}

FOR SELECTED CAUSES IN BRAZIL: A STUDY OF THE POSSIBLE IMPACT OF THE PANDEMIC BY THE NEW CORONAVIRUS

DOI: 10.16891/2317-434X.v10.e1.a2022.pp1191-1195

Recebido em: 22.04.2021 | Aceito em: 12.10.2021

\author{
Claudia Cristina de Aguiar Pereiraa, Carla Jorge Machadob \\ Departamento de Administração e Planejamento em Saúde da \\ Escola Nacional de Saúde Pública Sergio Arouca (ENSP) da Fiocruza \\ Universidade Federal de Minas Gerais ${ }^{b}$ \\ E-mail: carlajmachado@gmail.com
}

\section{RESUMO}

O estudo tem por objetivo comparar alguns indicadores relacionados à mortalidade no período anterior ao período inicial da pandemia de Covid-19 no Brasil. Trata-se de estudo observacional sobre internações, óbitos e letalidade hospitalar nos serviços próprios e conveniados ao SUS entre março e dezembro dos anos de 2019 e 2020 . Os dados foram obtidos nos Sistemas de Informações Hospitalares e Ambulatoriais do SUS e estratificados por meio da classificação correspondente aos Capítulos da Décima Revisão da Classificação Estatística Internacional de Doenças e Problemas Relacionados à Saúde (CID-10). Foram considerados, inicialmente, todos os Capítulos da CID-10, mas selecionados aqueles que apresentaram razão de letalidade 2020 em relação à 2019 igual ou superior a 1,5. Compararam-se os óbitos, internações e razões de taxa de letalidade entre 2019 e 2020 e, também, selecionaramse aqueles Capítulos cujos percentuais de letalidade foram diferentes entre si, ao nível de $5 \%$ de significância $(p<0,05)$. A maior parte dos óbitos e internações ocorreram pelas doenças do aparelho respiratório. As mortes referentes aos capítulos 8, 10, 13, 17 e 21 apresentaram razão de taxas de letalidade superiores à 1,5, sendo o capítulo 21 'Contatos com serviços de saúde' o que apresentou a maior razão. Os dados enfatizaram os diferenciais de letalidade no primeiro ano da pandemia em relação ao mesmo período no ano anterior à pandemia, especialmente em alguns grupos específicos de causas de morte, principalmente aqueles com forte interação com os serviços de saúde. Políticas públicas deverão enfocar as causas de mortalidade mais drasticamente alteradas pelo curso da pandemia.

Palavras-chave: Classificações em Saúde; SARS-CoV-2; Sistema Único de Saúde.

\section{ABSTRACT}

The study aims to compare a few indicators in the initial period of the Covid-19 pandemic with the same period in the year 2019 in Brazil. It is an observational account on hospitalizations, deaths and hospital fatality in the SUS's own and affiliated facilities between March and December of the years 2019 and 2020. The data were obtained from SUS Hospital and Outpatient Information Systems, and it was stratified through the classification corresponding to the Chapters of the Tenth Revision of the International Statistical Classification of Diseases and Related Health Problems (ICD-10). Initially, all the Chapters of the ICD-10 were considered, but those with a fatality ratio of 2020 in relation to 2019 equal to or greater than 1.5 were selected as well as those Chapters for which fatality proportions were different between periods at the $5 \%$ significance level $(p<0.05)$. Most deaths and hospitalizations occurred due to causes of the respiratory system. Deaths referring to chapters $8,10,13,17$ and 21 had a fatality rate higher than 1.5, and the Chapter 21 "Contacts with health services" had the highest ratio. The data emphasize the differences in case-fatality in the first year of the pandemic compared to the same period in the year before the pandemic, especially in some specific groups of causes of death, especially those with strong interaction with health services. Public policies should focus on the causes of mortality most drastically altered by the course of the pandemic.

Keyword: Health Classifications; SARS-CoV-2; Unified Health System. 
V.10 N.1 (2022) ISSN: 2317-434X

\section{INTRODUÇÃO}

A pandemia de Covid-19 foi decretada pela Organização Mundial da Saúde (OMS) em março de 2020 (OMS, 2020), e seus efeitos devastadores têm impactado o mundo em múltiplos setores. No Brasil, suas consequências, bem como sua longa duração comparativamente a outros países, perpassam aspectos econômicos, políticos, sociais, educacionais e de saúde (Tang et al., 2021). Lidar com o vírus SARS-CoV-2 e a doença por ele causada, trouxe inúmeros desafios ao sistema de saúde, desde a vigilância e atenção básica até os serviços de alta complexidade. Se, por um lado, tem havido pressão para tratar os acometidos pela infecção, por outro, prevenção, diagnóstico e tratamento a outras doenças tendem a ser postergados ou nunca oferecidos. Num contexto de escassez de recursos de saúde, como leitos, recursos humanos e outros insumos, a falta de diagnóstico e tratamento pode culminar em óbito.

O cenário atual exige um olhar cuidadoso sobre as mudanças trazidas pela pandemia no que diz respeito à mortalidade e alguns estudos vêm sendo divulgados sobre internações por Covid-19, excesso de mortes, mortalidade hospitalar e até sobre uso do registro civil para o estudo da mortalidade (Orellana et al., 2020; Silva et al., 2020). Entretanto, ainda é necessário investigar como as mudanças ocorridas durante a pandemia se dão ao nível dos grandes grupos de doenças em sua classificação tradicional. Assim, torna-se importante realizar esta avaliação através de indicadores relacionados ao cuidado, como o número de internações, óbitos e, também, para a letalidade à luz da classificação das doenças de forma mais abrangente. Desta forma, este estudo se propõe a comparar o período anterior à pandemia aos primeiros meses da pandemia, considerando importantes indicadores conforme as classificações de doenças em grandes grupos para elucidar diferenças que emergiram com a Covid-19.

\section{MÉTODOS}

Trata-se de estudo observacional que abrange internações, óbitos hospitalares, taxa de letalidade hospitalar - percentual de óbitos entre as internações - nos serviços próprios e conveniados ao SUS entre março e dezembro dos anos de 2019 e 2020. Os dados foram obtidos por busca nos Sistemas de Informações Hospitalares e Ambulatoriais do SUS (SIH) disponíveis no Datasus e, portanto, públicos e anônimos em conformidade com o artigo I da resolução 510/2016 da Comissão Nacional de Ética em Pesquisa. Os dados foram estratificados por meio da classificação correspondente aos Capítulos da Décima Revisão da Classificação Estatística Internacional de Doenças e Problemas Relacionados à Saúde (CID-10). Foram considerados, inicialmente, todos os Capítulos da CID-10, mas selecionados para apresentação aqueles que apresentaram razão de letalidade 2020 em relação à 2019 igual ou superior a 1,5 - letalidade em março a dezembro de 2020 em relação à letalidade em março a dezembro de 2019. Além do critério de razão, também, selecionaram-se aqueles Capítulos cujos percentuais de letalidade foram diferentes entre si ao nível de $5 \%$ de significância $(p<0,05)$ por meio de teste de comparação de proporções. Os programas Microsoft ${ }^{\circledR}$ Excel ${ }^{\circledR}$ for Mac 2011 e Stata/SE $®$ 12.0 for Mac foram utilizados para realizar a confecção das tabulações, gráficos e análises estatísticas.

\section{RESULTADOS E DISCUSSÃO}

A Tabela 1 e a Tabela 2 indicam os resultados encontrados, os quais se enquadraram em ambos os critérios de seleção dos Capítulos.

Tabela 1 - Comparativo de óbitos, internações e letalidade para Capítulos selecionados da CID-10, Brasil 2019 e 2020

\begin{tabular}{|c|c|c|c|c|c|c|}
\hline \multirow[b]{2}{*}{ Capítulo e Título do Capítulo } & \multicolumn{2}{|l|}{ Óbitos } & \multicolumn{2}{|c|}{ Internações } & \multicolumn{2}{|c|}{ Letalidade (\%) } \\
\hline & 2019 & 2020 & 2019 & 2020 & 2019 & 2020 \\
\hline Cap. 8 - Doenças do ouvido e apófise mastóide & 33 & 22 & 18.333 & 8.148 & 0,2 & 0,3 \\
\hline Cap. 10 - Doenças do aparelho respiratório & 83.612 & 76.685 & 1.037 .370 & 594.919 & 8,1 & 12,9 \\
\hline Cap. 13 - Doenças do sist osteomus/tec conjuntivo & 1.098 & 991 & 186.102 & 110.111 & 0,6 & 0,9 \\
\hline Cap. 17 - Malformações cong. e anomalias cromossômicas & 1.805 & 1.699 & 77.137 & 47.194 & 2,3 & 3,6 \\
\hline Cap. 21 - Contatos com serviços de saúde & 1.021 & 1.086 & 232.045 & 152.958 & 0,4 & 0,7 \\
\hline
\end{tabular}


V.10 N.1 (2022) ISSN: $2317-434 \mathrm{X}$

Tabela 2 - Razões de óbitos, de internações e letalidade entre 2019 e 2020 para Capítulos selecionados da CID-10, Brasil

\begin{tabular}{|c|c|c|c|}
\hline Capítulo/Título do Capítulo & óbitos & internacões & letalidade \\
\hline Cap 08 - Doenças do ouvido e da apófise mastóide & 0,67 & 0,45 & 1,5 \\
\hline Cap10 - Doenças do aparelho respiratório & 0,92 & 0,58 & 1,6 \\
\hline Cap 13 - Doenças sist osteomuscular e tec conjuntivo & 0,90 & 0,59 & 1,5 \\
\hline Cap 17 - Malformações congênitas deformidades e anomalias cromoss & 0,94 & 0,61 & 1,5 \\
\hline Cap 21 - Contatos com serviços de saúde & 1,06 & 0,66 & 1,6 \\
\hline
\end{tabular}

A maior parte dos óbitos e internações ocorreram pelas doenças do aparelho respiratório (Tabela 1). Quanto aos óbitos, ocorreram 83,6 e 76,7 mil óbitos em 2019 e em 2020, respectivamente. As internações foram de 1,03 milhão em 2019 e 590,9 mil em 2020. As taxas de letalidade foram, respectivamente, $8,1 \%$ e $12,9 \%$ em 2019 e em 2020. Em seguida, quanto às malformações congênitas (Capítulo 17) no que se refere aos óbitos, foram de 1,8 e 1,7 mil em 2019 e 2020 respectivamente, com 77,1 e 47,1 internações em 2019 e 2020, respectivamente. A letalidade passou de 2,3\% para 3,6\%. Doenças referentes aos Contatos com serviço de saúde (Capítulo 21) responderam por 1,0 e 1,1 mil óbitos em 2019 e 2020 , respectivamente, e por 232,0 e 153,0 mil internações em 2019 e 2020, respectivamente. Outras doenças que tiveram aumento da letalidade, com queda nos óbitos, mas redução ainda maior nas internações foram as do Capítulo 13 (Doenças do sistema osteomuscular e tecido conjuntivo) e 8 (Doenças do ouvido e da apófise mastoide) (Tabela 1). Os achados do presente estudo corroboram pesquisa de Normando et al. (2021) que estudaram doenças cardiovasculares. A pesquisa indicou que, embora o número absoluto de mortes hospitalares tenha diminuído em 2020 frente a 2019, a letalidade hospitalar de internações por doenças cardiovasculares aumentou (Normando et al., 2021). Uma das razões apontadas para esse aumento da letalidade se refere ao atraso na procura de atendimento médico e internações de pacientes com quadros mais graves (Normando et al., 2021).

A Tabela 2 traz as razões e auxilia uma análise mais completa do panorama pré e pós-pandemia. Chama a atenção o aumento da letalidade por causas relacionadas ao Capítulo 21, que foi de $61 \%$. De fato, houve um acréscimo de $6 \%$ no número de óbitos assim classificados, acompanhado por um decréscimo de $34 \%$ nas internações por esse motivo (razões de 1,06 e 0,66, respectivamente). É possível que casos que tenham sido classificados neste capítulo tenham chegado aos serviços de saúde mais tardiamente em 2020 comparativamente a 2019 e, portanto, com menos possibilidade de ação para prevenção do óbito. É importante lembrar que os códigos $Z$ das categorias Z00-Z99 são fornecidas para ocasiões em que circunstâncias diferentes de doença, lesão ou causa externa classificáveis nas categorias A00-Y89 são registradas como 'diagnósticos' ou 'problemas' (ICD.Codes, 2021). Isso pode surgir de duas maneiras principais: (a) Quando uma pessoa que pode ou não estar doente utiliza os serviços de saúde para algum propósito específico, como receber cuidados ou serviços limitados para uma condição atual, doar um órgão ou tecido, para receber vacinação profilática (imunização), ou para discutir um problema que em si não é uma doença ou lesão. (b) Quando alguma circunstância ou problema está presente que influencia o estado de saúde da pessoa, mas não é em si uma doença ou lesão atual (ICD.Codes, 2021). É possível que o deslocamento do atendimento para casos relacionados à Covid-19 tenha gerado escassez de profissionais, menos internações, sendo estas restritas aos casos mais graves e com pouca capacidade de resolução do caso. Cabe observar que, dentre as causas elencadas, os contatos por serviço de saúde foram as únicas que responderam pelo aumento no número de óbitos. Nesse sentido, Teixeira et al. (2020) analisaram o afastamento do trabalho por parte dos trabalhadores da saúde que aumentou na pandemia em relação a períodos anteriores, e, dessa forma, pode-se inferir ser esta mais uma razão para o atraso nos atendimentos, desta vez pelo fato dos pacientes não conseguirem ter atendidas suas demandas.

Além disso, houve um aumento da letalidade por doenças do Capítulo 10 - 60\% a mais comparado a 2020 - com queda de $43 \%$ no número de internações, mas de menor magnitude dos óbitos (-8\%). Bronquites, bronquiolites, laringites, traqueítes agudas, doenças do nariz e dos seios paranasais, além de infecções das vias aéreas superiores estiveram entre as principais causas elencadas neste capítulo (resultados não mostrados), indicando a possibilidade de que algumas causas de 


\section{V.10 N.1 (2022) ISSN: 2317-434X}

internação e óbitos relacionadas à Covid-19 tenham sido inicial - e incorretamente - classificadas nesse capítulo; outra hipótese é que a gravidade desses casos tenha sido muito maior do que em 2020.

Quanto ao Capítulo 13, as cifras das razões foram muito próximas as das doenças respiratórias. Cabe observar que a artrose, transtornos do tecido mole e outros transtornos articulares não classificados, nesta ordem, foram as doenças com maior letalidade, sendo a queda nas internações muito mais visíveis do que a queda nos óbitos de um ano para outro, sendo que no caso dos tecidos moles houve aumento do número de óbitos com queda nas internações, sugerindo maior gravidade nas internações em 2020. Já as malformações - Capítulo 17 - seguiram padrão semelhante, sugerindo a mesma tendência na gravidade dos casos em 2020. No caso da fenda labial e palatina, que faz parte deste capítulo e teve aumento de 7 para 9 óbitos (dados não mostrados), com queda nas internações à metade de um ano para outro, mostra-se claramente evidência de atraso na intervenção correcional. De fato, segundo Sousa e Roncalli (2021), a escassez de recursos de saúde faz com que o tratamento cirúrgico primário esteja fora do alcance de milhares de crianças que residem em países em desenvolvimento. Segundo os autores, esses aspectos podem levar ao acréscimo de taxas de mortalidade neonatal por complicações relacionadas à desnutrição e às infecções em crianças nascidas com fissuras labiopalatinas mais graves (Sousa; Roncalli, 2021).

No caso das doenças do ouvido, apesar de queda nos óbitos e de numericamente serem em número bem inferior ao das demais causas, a letalidade aumentou em níveis considerados significativos pelo teste de diferença de proporções $(\mathrm{p}<0,05)$. A queda no número de internações superou os 50\% em relação a 2019.

\section{CONCLUSÕES}

O trabalho apontou alterações nos níveis de internações, óbitos e letalidade desencadeadas a partir da pandemia de COVID-19. O estudo ressaltou os principais capítulos da CID-10 afetados no período anterior e imediatamente posterior ao início da pandemia no Brasil.

Cinco capítulos da CID-10 tiveram destaque, com aumento da letalidade pelas causas de morte neles contidas de pelo menos 50\%. Os achados também refletem redução das internações por estas mesmas causas. Por exemplo, o aumento da letalidade por causas relacionadas ao Capítulo 21 referente aos contatos com serviços de saúde, foi de $61 \%$, com aumento de $6 \%$ no número de óbitos assim classificados e decréscimo de $34 \%$ nas internações.
Por outro lado, algumas doenças apresentaram aumento da letalidade, com queda nos óbitos e redução ainda maior nas internações (Capítulos 13 e 8 referentes a doenças do sistema osteomuscular e tecido conjuntivo e doenças do ouvido e da apófise mastoide). Tais achados podem refletir eventos de internação menos frequentes por dificuldades de acesso aos serviços de saúde, quer pela diminuição da oferta ou por medo dos usuários, porém com mais complicações que resultam em óbitos, as quais se refletem na letalidade. Estes achados estão em consonância com estudos realizados no Brasil e no exterior. Um trabalho realizado nos Estados Unidos, identificou reduções nas internações e apontou como possíveis causas o medo de contaminação dos pacientes quanto aos ambientes hospitalares, mudanças no comportamento dos profissionais da saúde ao prescrever internações e alterações comportamentais dos usuários de serviços de saúde (Blecker et al., 2020). Outro estudo, realizado no Brasil, comparou a evolução das internações em seis capitais brasileiras fortemente afetadas pela pandemia, identificando queda significativa no número de internações a partir de março de 2020, com o primeiro pico de internações por COVID-19 em maio de 2020. Nas seis capitais, observaram-se significativas reduções no número médio de hospitalizações por quinzena desde o início da pandemia. A proporção de internações cirúrgicas e eletivas caiu significativamente em todas as capitais estudadas, enquanto a proporção de internações com o uso de UTI aumentou significativamente. Aumentos significativos na mortalidade hospitalar também foram registrados nas seis capitais com a pandemia, incluindo ou excluindo hospitalizações COVID-19 da comparação (Portela et al., 2021).

Em geral, a redução nas internações, principalmente nas cirúrgicas e eletivas, era esperada, já que a situação emergencial imposta pela pandemia impôs a reorganização dos fluxos de atendimento, a realocação de mão de obra e recursos tecnológicos disponíveis em hospitais. Além disso, exigiu a incorporação de novos recursos, como a abertura de hospitais campanha para atendimento exclusivo de paciente com Covid-19 (Portela et al., 2021).

Nos casos em que a mortalidade hospitalar aumentou no período da pandemia comparativamente ao período anterior, pode-se suspeitar de situações em que os pacientes simplesmente foram impossibilitados de conseguir vagas de internações devido ao grande volume de pacientes internados com Covid-19. Estudos sobre filas de espera e tempo desde a solicitação até a internação nos sistemas de regulação poderão elucidar o que de fato ocorreu na ponta durante os meses da pandemia.

No que concerne às causas contidas no Capítulo 


\section{V.10 N.1 (2022) ISSN: $2317-434 X$}

13 (doenças do sistema osteomuscular e tecido conjuntivo), o número absoluto de óbitos apresentou pouca variação entre os períodos, mas a redução no número de internações pode estar refletindo diminuições no número de fraturas, acidentes de trânsito, postergamento de cirurgias eletivas ou opção por tratamentos mais conservadores e não cirúrgicos. (Lima et al., 2020). Ainda assim, é necessária atenção para o aumento significativo da letalidade, que já foi verificada em outros estudos (Normando et al, 2021; Wolf et al, 2020).
As limitações do estudo devem ser mencionadas. Primeiramente, trata-se de estudo natureza observacional e, portanto, não é possível inferir causalidade entre a pandemia e os indicadores. O uso dos SIH e SIA/SUS também impossibilita inferências e análises para os beneficiários de planos privados de saúde que não utilizaram a rede do SUS para seus tratamentos. Desta foram, não é possível apreender o cenário completo das internações e mortalidade no Brasil. Contudo, ainda com as lacunas inerentes ao trabalho, trata-se de ponto de partida para a análise do possível impacto da pandemia sobre indicadores de saúde.

\section{REFERÊNCIAS}

Blecker S, Jones SA, Petrilli CM, Admon AJ, Weerahandi H, Francois F, Horwitz LI. Hospitalizations for chronic disease and acute conditions in the time of COVID-19. JAMA Intern Med. 2020; 181(2):269-271. doi:10.1001/jamainternmed.2020.3978.ICD.Codes. https://icd.codes/icd10cm/chapter21/Z40-Z53

Lima EBS, Belangero PS, Falótico GG, Mansur NSB, Luzo MVM, Reis FB. Intervention protocol of the Orthopedics and Traumatology Department of a highcomplexity university hospital to cope with the COVID19 pandemic. Rev Bras Ortop (Sao Paulo). 2020; 55(3):269-277. doi: 10.1055/s-0040-1712972.

OMS (2020). WHO Director-General's opening remarks at the media briefing on COVID-19 - 11 March 2020. Disponível em: https://www.who.int/directorgeneral/speeches/detail/who-director-general-s-openingremarks-at-the-media-briefing-on-covid-19---11-march2020. Acesso em 20/04/2021.

Normando P, Araújo-Filho JA, Fonseca G, Rodrigues R, Oliveira V, Hajjar L, Almeida AL, Bocchi E, Salemi V, Melo M. Redução na Hospitalização e Aumento na Mortalidade por Doenças Cardiovasculares durante a Pandemia da COVID-19 no Brasil. Arq. Bras. Cardiol. [online]. 2021; doi: 10.36660/abc.20200821.

Orellana J, Cunha G, Marrero L, Moreira R, Leite I, Horta B. Excesso de mortes durante a pandemia de COVID-19: subnotificação e desigualdades regionais no Brasil. Cad. Saúde Pública 2020; 36(1):e00259120. doi: 10.1590/0102-311X00259120.

Portela C, Pereira C, Lima S, Andrade C, Martins M.
Patterns of hospital utilization in the Unified Health System in six Brazilian capitals: comparison between the year before and the first six first months of the COVID-19 pandemic. BMC Health Serv Res 2021; 21:976. doi:10.1186/s12913-021-07006-X.

Silva G, Jardim B, dos Santos C. Excesso de mortalidade no Brasil em tempos de COVID-19. Ciênc. saúde coletiva 2020; 25(9). doi: 10.1590/1413-81232020259.23642020.

Sousa G, Roncalli A. Fatores associados ao atraso no tratamento cirúrgico primário de fissuras labiopalatinas no Brasil: uma análise multinível. Ciênc. saúde coletiva 2021; 26(2): 3505-3515. doi: 10.1590/141381232021269.2.23592019.

Tang Y, Serdan T, Alecrim A, Souza D, Nacano B, Silva F, Silva E, Poma S, Gennari-Felipe M, Iser-Bem P, Masi L, Tang S, Levada-Pires A, Hatanaka E, Cury-Boaventura M, Borges F, Pithon-Curi T, Curpertino M, Fiamoncini J, Leandro C, Gorjao R, Curi R, Hirabara S. A simple mathematical model for the evaluation of the long first wave of the COVID-19 pandemic in Brazil. Sci Rep 2021; 11:16400. doi:10.1038/s41598-021-95815-9

Teixeira CFS, Soares CM, Souza EA, Lisboa ES, Pinto ICM, Andrade LR, Espiridião MA. A saúde dos profissionais de saúde no enfrentamento da pandemia de Covid-19. Ciênc. saúde coletiva 2020, 25(9):3465-3474. doi: 10.1590/1413-81232020259.19562020.

Woolf SH, Chapman DA, Sabo RT, Weinberger DM, Hill L. Excess Deaths From COVID-19 and Other Causes. JAMA 2020, 324(5):510-513. 